Apidologie, 1984, 15 (2), 171-188

\title{
FAKTOREN, DIE DIE BILDUNG VON HYDROXYMETHYLFURFURAL IM HONIG BEEINFLUSSEN
}

\author{
Franz Josef PICHLER, Günther VORWOHL und Karlheinz GIERSCHNER \\ Landesanstalt fïr Bienenkunde und Institut für Lebensmitteliechnologie \\ Universität Hohenheim, D 7000 Stuttgart 70
}

\section{ZUSAMMENFASSUNG}

Hydroxymethylfurfural (HMF) ensteht, wenn Honig erhitzt oder in warmen Räumen gelagert wird. Hohe HMF-Werte zeigen unerwünschte Reaktionen im Honig an : Aromaverluste, Aromaveränderungen, Verlust an bakteriostatischer Wirkung. Die Qualitätsstandards vieler Länder geben höchstzulässige Werte für HMF an. Es ist daher von praktischem Interesse, die Faktoren (pH-Wert, Zuckerart, Konzentration, Höhe der Temperatur und Einwirkungszeit, Vorhandensein von Aminosäuren) zu prüfen, die Einfluß auf den HMF-Gehalt des Honigs nehmen. In der vorliegenden Arbeit wird mit vereinfachten Honigmodellen (Zucker plus Citrat-Phophat-Puffer) gearbeitet. Die HMF-Messsung erfolgte nach der photometrischen Methode von WinkLER..

\section{EINLEITUNG}

\section{Problemstellung und Allgemeines}

Die Messung des Hydroxymethylfurfural (HMF)-Gehalts dient der Kontrolle von Wärmeeinwirkungen auf den Honig, denen dieser bei der Gewinnung, Klärung und Lagerung, beim Transport sowie bei der Abfüllung und Vermarktung ausgesetzt sein kann. Dabei dient der HMF-Gehalt als Indikator für andere unerwünschte wärmebedingte Reaktionen des Honigs : Aromaverluste und Aromaveränderungen, Verminderung der enzymatischen Aktivität und der bakteriziden Eigenschaften, Bräunungsreaktionen u.s.w.

Toxikologisch ist das HMF für den Menschen unbedenklich. Dies wurde von LANG et al. (1971) in einer ausführlichen Arbeit dargelegt. Hingegen wirken erhitzter Honig und andere erhitzte Zuckerprodukte leicht toxisch auf die Honig- 
biene, wobei neben dem HMF auch noch andere Abbauprodukte der Zucker eine Rolle spielen mögen (Temnov, 1950, Rapeanu, 1969, Jachimowicz und Ruttner, 1974, Jachimowicz und El Sherbini, 1975). Erhitzter Honig und andere erhitzte Zuckerprodukte sollten daher nicht als Winterfutter verwendet werden und bei kleinen Bieneneinheiten, wie z.B. Völkchen in Begattungskästchen, auch nicht in der warmen Jahreszeit verfüttert werden.

Die Erfahrung lehrt, daß die verschiedenen Honige bei einer gleichartigen Wärmebehandlung unterschiedliche HMF-Mengen bilden. Die Ursachen sind in der wechselnden chemischen Zusammensetzung der verschiedenen Honigarten zu suchen. In jedem Honig gibt es dabei fördernde und hemmende Faktoren. Da der HMF-Gehalt bei der Beurteilung der Honigqualität herangezogen wird und die Gesetzgeber höchstzulässige Werte festgesetzt haben, besteht ein erhebliches praktisches Interesse, die Faktoren zu kennen, die die HMF-Bildung bzw. die messbare HMF-Menge beeinflussen.

In der vorliegenden Arbeit wird mit Modell-Lösungen gearbeitet, die sich an der Zusammensetzung natürlicher Honige orientieren. Dies gestattet eine klarere Herausarbeitung der Faktoren, die den messbaren HMF-Gehalt steuern.

\section{MATERIAL UND METHODIK}

Die Modelle sind Zuckerlösungen in einem Dinatriumhydrogenphosphat-Citronensäure-Puffer mit einem pH-Wert von 4,2 . Dieser pH-Wert kann als guter Mittelwert der in Honigen vo:liegenden Wasserstoffionenkonzentrationen angesehen werden.

Die Modell-Lösungen enthalten in $100 \mathrm{~g} 0,1078 \mathrm{~g} \mathrm{Na}^{+}, 0,2249 \mathrm{~g} \mathrm{HPO}_{4}^{2-}$ und $0,174 \mathrm{~g}$ Citronensäure.

Die folgenden Reagenzien wurden für die Ansätze verwendet :

D-Glucose, Merck Art. 8342;

D-Fructose, Merck Art. 5321;

Maltose, Merck Art. 5910 ;

$\alpha$ D-Melezitose $\times 2 \mathrm{H}_{2} \mathrm{O}$, Serva 28550 ;

Saccharose reinst, DAB 7, Merck Art. 7653 ;

Raffinose, Merck Art. 7549 ;

L-AIanin Merck Art. 1007 ;

L-Asparaginsäure, Merck Art. 126 ;

L-Glutaminsäure, Merck Art. 291 ;

L-Serin, Merck Art. 7769 ;

L-Phenylalanin, Merck Art. 7256;

L-Prolin, Merck Art. 7434 ;

di-Natriumhydrogenphosphat-12-hydrat, Merck Art. 6579 ;

Citronensäure, Merck Art. 244.

Bei der Erhitzung wurde, wenn nicht anders angegeben, so verfahren, da $B$ die Modelle in einem Wärmeschrank mit Luftumwälzung Temperaturen von $45^{\circ} \mathrm{C}, 65^{\circ} \mathrm{C}$ und $85^{\circ} \mathrm{C}$ ausgesetzt wurden. Alle 10 Stdn. wurde der HMF-Gehalt bestimmt. Bei jeder Messung blieb die Modell-Lösung bei Labortemperatur 1 Std. stehen. 
Die Messung der HMF-Gehalts erfolgte nach dem photometrischen Verfahren von WiNkLER (1955). Dabei wurden die von VorwoHL (1969) empfohlenen Kautelen eingehalten.

Als Messgerät stand das Spektralphotometer Typ PM 2 DL der Firma Zeiss zur Verfügung. Die angegebenen HMF-Werte wurden aus 3 Messwerten gemittelt.

Beim Bereiten der Toluidin-Lösung beobachtet man eine Gelbfärbung mit jedoch stets unterschiedlicher Intensität. Diese Verfärbung ist die Folge einer Oxidation des Amins. Auch feste Amine sind empfindlich gegenüber Luftoxidation, wenn auch weniger als die flüssigen, wie z.B. das Anilin. Reine Präparate zersetzen sich nicht so rasch wie technische Produkte. Es wurde daher stets reines Toluidin (Smp. $45^{\circ} \mathrm{C}$ ) verwendet (Merck Art. 808315, inzwischen ersetzt durch Art. 10841).

\section{VERSUCHE UND ERGEBNISSE}

I. HMF-Bildung in Abhängigkeit von der chemischen Natur der verwendeten Zucker und ihrer Konzentration

\section{Allgemeines}

Erwärmung auf $45{ }^{\circ} \mathrm{C}$ bis zu 50 Stunden entspricht einer schonenden Behandlung. Längere Erwärmungen im Bereich um $65^{\circ} \mathrm{C}$ können sich in der Praxis ergeben, wenn man versucht, größere Mengen festkandierten Honigs möglichst kurzfristig zu verflüssigen. Ferner ergibt sich bei der Filterung des Honigs die Notwendigkeit, für längere Zeit höhere Temperaturen einzuhalten. Zur Erzielung besonders klaren Honigs wird vor allem im angelsächsischen Raum der Honig durch Diatomeenerde (Kieselgur) oder ähnliche feinkörnige Materialien gepreßt.

Durch Erhitzung auf Temperaturen um $85^{\circ} \mathrm{C}$ kann man Wachs und Honig voneinander trennen. Als Methode zur Gewinnung des Honigs aus der Wabe ist das Verfahren auf Gebiete mit traditioneller Bienenzucht in Beuten ohne bewegliche Rähmchen beschränkt, die nicht geschleudert werden können. Es gibt aber auch andere, schonende Verfahren zur Gewinnung des Honigs (Pressen, Austropfen). Das Ausschmelzen muß also nicht zwangsläufig erfolgen.

In modernen Großbetrieben, besonders in Australien, wird das Ausschmelzverfahren zur Abtrennung des Honigs aus dem Entdecklungswachs verwendet. Auch hier gibt es Verfahren (Zentrifugieren in Spezialschleudern), bei denen der Honig keiner Wärmebelastung ausgesetzt wird.

\section{Fructose}

Die Abb. 1 zeigt die Abhängigkeit der HMF-Bildung von der Fructosekonzentration bei $65^{\circ} \mathrm{C}$. Die HMF-Bildung nimmt erwartungsgemäß mit steigender Erwärmungszeit zu und auch zunächst mit steigender Fructosekonzentration. Dies gilt jedoch nur für Konzentrationen bis $50 \%$. Bei $60 \%$-igen Lösungen geht 


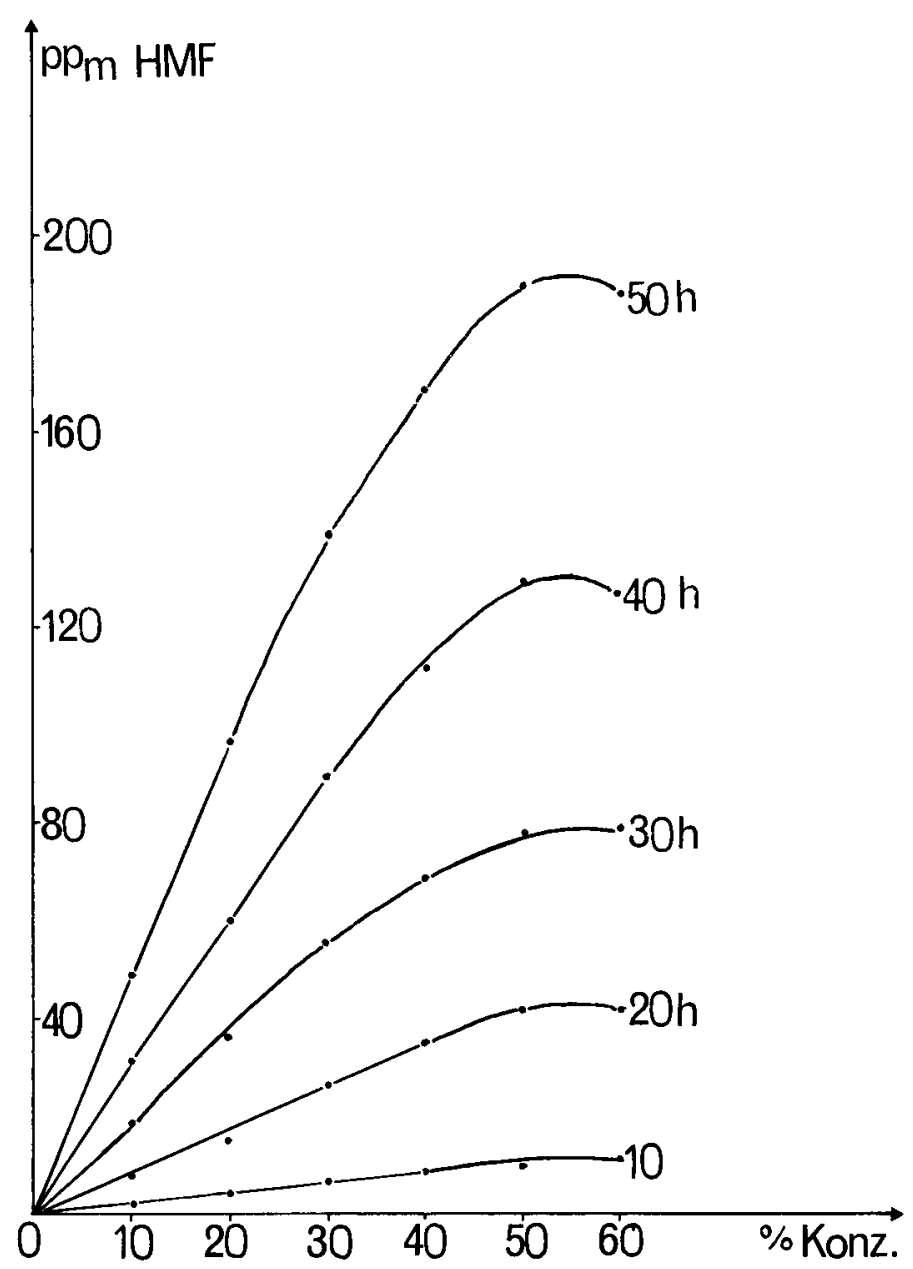

Авв. 1. - HMF-Bildung bei Fructose in Abhängigkeit von der Konzentration und der Zeit Fig. 1. - HMF production in fructose-solutions showing dependence on concentration and time

der HMF-Gehalt zurück, was auf ein Weiterreagieren im Rahmen der MaillardReaktionen hindeutet (Baumann und Gierschner, 1974). Die Abb. 2 bringt die Ergebnisse einer weiteren Versuchsreihe, diesmal mit Konzentrationen bis zu $80 \%$ Fructose bei Temperaturen von $85^{\circ} \mathrm{C}$. Hier, in den hochkonzentrierten Lösungen, zeigt sich der Rückgang des HMF-Gehalts nach Erreichen des Maximums noch deutlicher. 


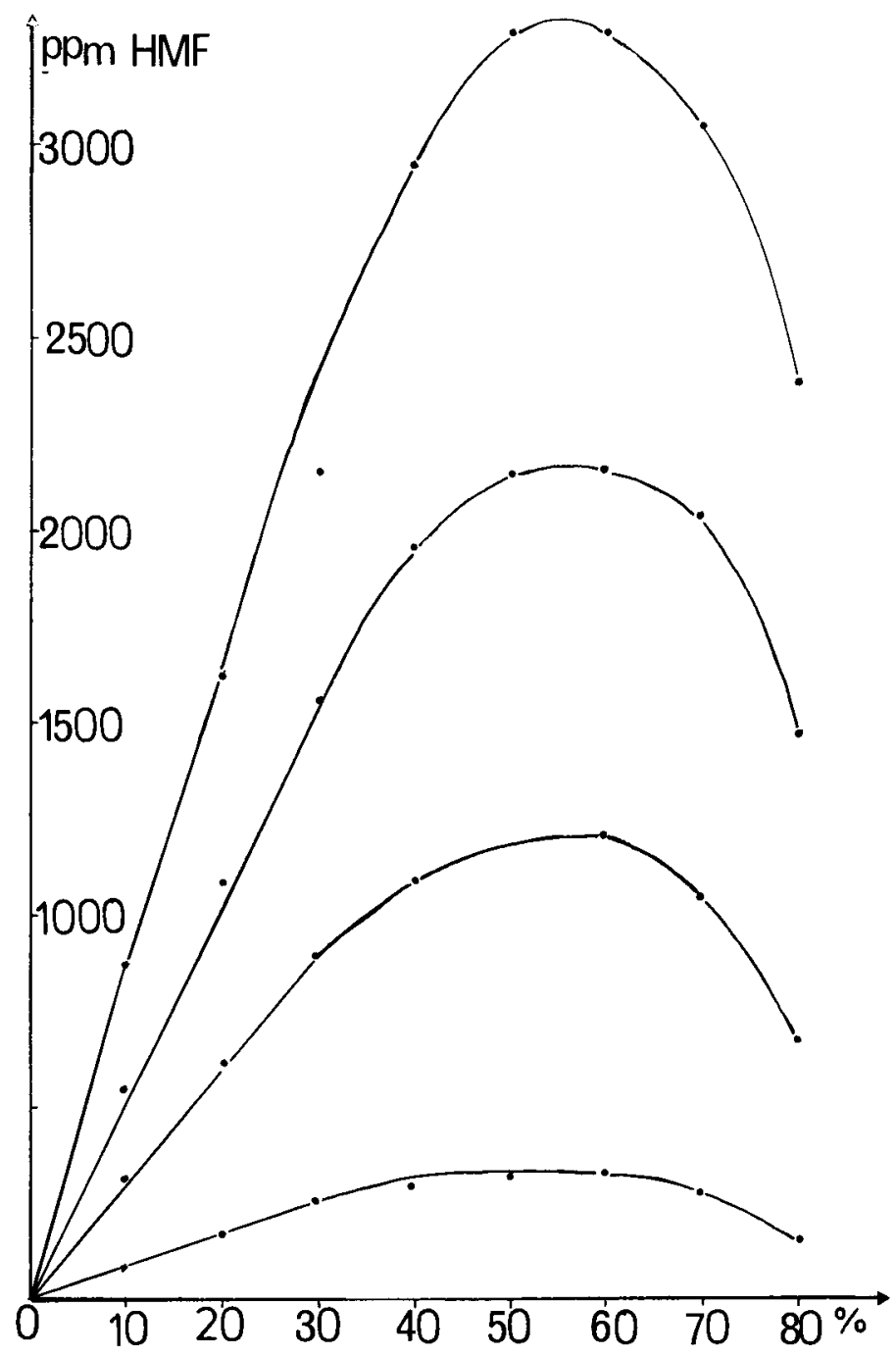

Авв. 2. - HMF-Bildung bei Fructose in Abhängigkeit von der Konzentration und der Zeit bei $85^{\circ} \mathrm{C}$

Die Kurven entsprechen von unten nach oben Erhitzungszeiten von 10, 20, 30 und 40 Stunden

FIG. 2. - The dependence of HMF production in fructose-solutions on concentration and time at $85^{\circ} \mathrm{C}$

The curves correspond to heating times of (from bottom to top) 10, 20,30 and 40 hours

\section{Glucose}

Die Kurvenschar der Abb. 3 gibt die Ergebnisse einer Versuchsreihe mit Glucoselösungen wieder. 


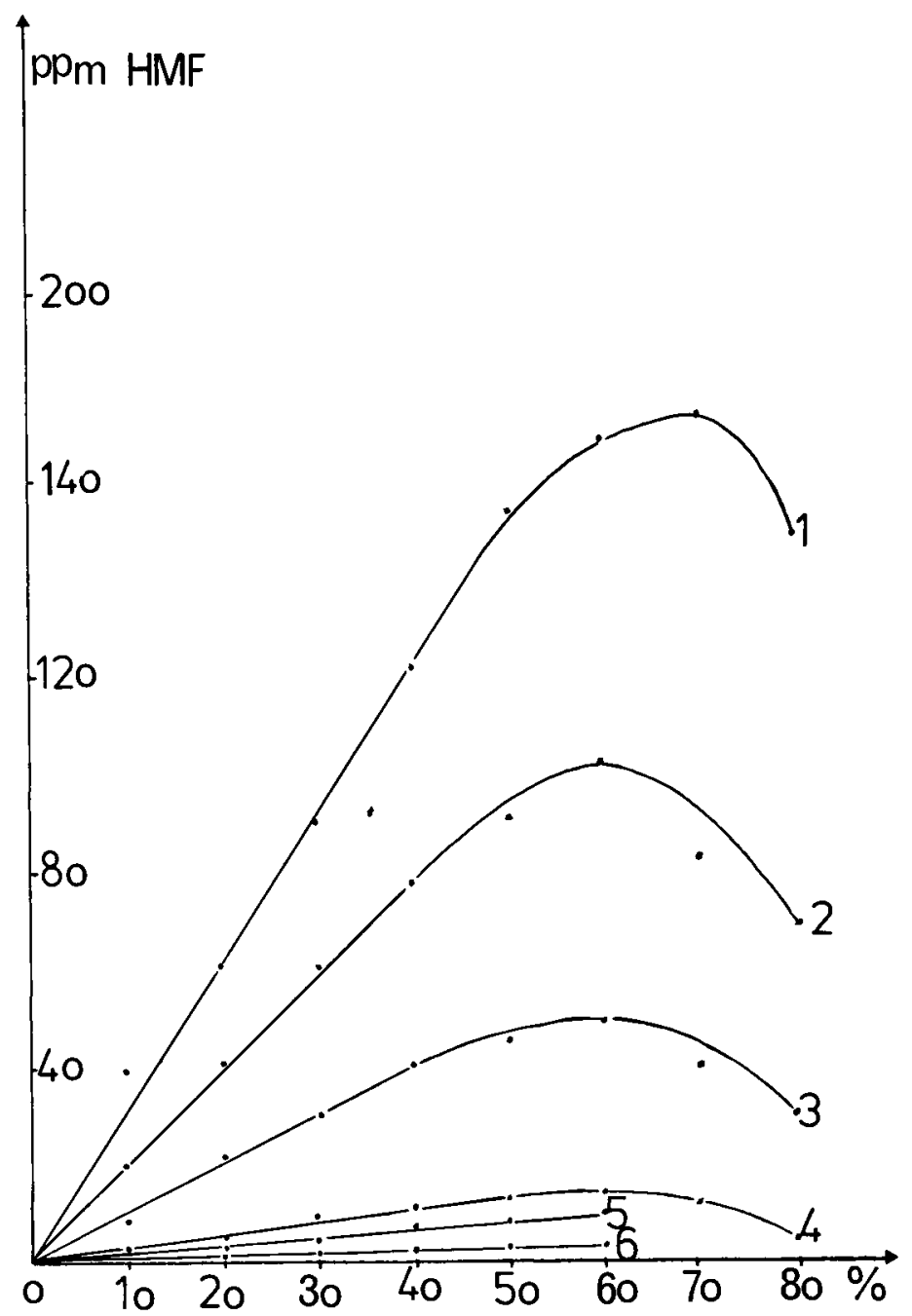

ABB. 3. - HMF-Bildung bei Glucose in Abhängigkeit von der Konzentration, der Temperatur und der Zeit

$$
\begin{array}{llll}
1=40 \mathrm{~h} & 85^{\circ} \mathrm{C} & 2=30 \mathrm{~h} & 85^{\circ} \mathrm{C} \\
3=20 \mathrm{~h} & 85^{\circ} \mathrm{C} & 4=10 \mathrm{~h} & 85^{\circ} \mathrm{C} \\
5=50 \mathrm{~h} & 65^{\circ} \mathrm{C} & 6=10 \mathrm{~h} & 65^{\circ} \mathrm{C}
\end{array}
$$

FIG. 3. - The dependence of HMF production in glucose-solutions on concentration, temperature and time.

Die HMF-Bildung erfolgt erheblich langsamer als bei der Fructose. In $40 \%$-iger Lösung bilden sich z.B. aus Fructose in 40 Stunden bei $85^{\circ} \mathrm{C}$ 
2950 ppm HMF, bei der Glucose sind es nur 114 ppm. Die Abhängigkeit der HMF-Bildung von der Konzentration der Lösung entspricht weitgehend dem Verhalten der Fructose.

\section{Fructose und Glucose bei niedriger Temperatur}

Die HMF-Entstehung in Glucose- und Fructoselösungen bei der niedrigsten Versuchstemperatur $\left(45^{\circ} \mathrm{C}\right)$ geht aus der Tab. 1 hervor. In den graphischen Darstellungen sind die niedrigen Werte schwer abzulesen.

TAB. 1. - Gebildete HMF-Menge in Glucose- und Fructoselösungen in Abhängigkeit von der Zeit und der Konzentration bei $45^{\circ} \mathrm{C}$

TABL. 1. - HMF in glucose and fructose solutions and its dependence on the time and the concentration at $45^{\circ} \mathrm{C}$

\begin{tabular}{|c|c|c|c|c|c|c|}
\hline \multirow{3}{*}{$\begin{array}{c}\text { Konzentration } \\
\text { Concentration } \\
\text { in } \%\end{array}$} & \multicolumn{6}{|c|}{$\begin{array}{l}\text { HMF (ppm) nach } \\
\text { HMF (ppm) after }\end{array}$} \\
\hline & \multicolumn{2}{|c|}{8} & \multicolumn{2}{|c|}{13} & \multicolumn{2}{|c|}{$\begin{array}{l}21 \text { Tagen } \\
21 \text { days }\end{array}$} \\
\hline & Glucose & Fructose & Glucose & Fructose & Glucose & Fructose \\
\hline 10 & 0 & 5 & 1 & 18 & 2 & 56 \\
\hline 20 & 0 & 16 & 2 & 39 & 5 & 111 \\
\hline 30 & 0 & 21 & 3 & 55 & 7 & 151 \\
\hline 40 & 0 & 26 & 4 & 69 & 9 & 184 \\
\hline 50 & 0 & 25 & 4 & 75 & 9 & 212 \\
\hline 60 & 0 & 27 & 5 & 82 & 11 & 230 \\
\hline
\end{tabular}

\section{Fructose und Glucose in Mischung}

Mischt man Modell-Lösungen aus Fructose und Glucose (Tab. 2), so nimmt erwartungsgemäß die gebildete HMF-Menge mit fallendem Fructosegehalt ab. Der niedrige Wassergehalt $(20 \%)$ des Modells bedingt offensichtlich auch eine Abnahme der HMF-Bildung. $40 \%$-ige Fruchtzuckerlösung bildet z.B. für sich alleine bei $65^{\circ} \mathrm{C}$ und Standard-pH 4,2 in 40 Stunden $112 \mathrm{ppm}$ HMF. In der Glucoselösung werden bei identischem Versuchsansatz 5 ppm HMF gemessen. Die Modell-Lösungen mit je $50 \%$ Glucose und Fructose in der Trockenmasse und $20 \%$ Pufferlösung liefern in 40 Stunden und bei $65^{\circ} \mathrm{C}$ nur 34 ppm HMF.

6. Abhängigkeit der HMF-Bildung aus Fructose und Glucose von der Zeit

Die HMF-Bildung ist zeitabhängig. Es wird jedoch in den gleichen Zeiträumen bei sonst gleichen Bedingungen nicht immer die gleiche HMF-Menge gebildet. 
TAB. 2. - Abhängigkeit der HMF-Bildung vom pH-Wert der Fructose-Glucosekonzentration und der Zeit bei $65^{\circ} \mathrm{C}$

TABL. 2. - Dependence of HMF production on $p H$ value, on fructose-glucose concentration and time at $65^{\circ} \mathrm{C}$

\begin{tabular}{|c|c|c|c|c|c|}
\hline \multirow{2}{*}{$\begin{array}{c}\text { Versuchsreihe } \\
\text { Trial }\end{array}$} & \multirow{2}{*}{$\begin{array}{c}\text { Zuckerkon- } \\
\text { zentration } \\
\text { Sugar } \\
\text { concentration } \\
(\%)\end{array}$} & \multirow{2}{*}{$\begin{array}{l}\mathrm{pH}-\text { Wert } \\
\mathrm{pH} \text { value }\end{array}$} & \multicolumn{3}{|c|}{$\begin{array}{l}\text { HMF (ppm) nach } \\
\text { HMF (ppm) after }\end{array}$} \\
\hline & & & $20 \mathrm{~h}$ & $40 \mathrm{~h}$ & $60 \mathrm{~h}$ \\
\hline 1 & $\begin{array}{l}10 \mathrm{G} \\
90 \mathrm{~F}\end{array}$ & $\begin{array}{l}6,2 \\
4,2 \\
2,2\end{array}$ & $\begin{array}{r}2 \\
11 \\
271\end{array}$ & $\begin{array}{r}5 \\
54 \\
465\end{array}$ & $\begin{array}{c}13 \\
110 \\
710\end{array}$ \\
\hline 2 & $\begin{array}{ll}20 \mathrm{G} \\
80 \mathrm{~F}\end{array}$ & $\begin{array}{l}6,2 \\
4,2 \\
2,2\end{array}$ & $\begin{array}{r}2 \\
12 \\
195\end{array}$ & $\begin{array}{r}5 \\
48 \\
394\end{array}$ & $\begin{array}{r}11 \\
107 \\
590\end{array}$ \\
\hline 3 & $\begin{array}{l}30 \mathrm{G} \\
70 \mathrm{~F}\end{array}$ & $\begin{array}{l}6,2 \\
4,2 \\
2,2\end{array}$ & $\begin{array}{r}2 \\
12 \\
166\end{array}$ & $\begin{array}{r}4 \\
46 \\
292\end{array}$ & $\begin{array}{r}10 \\
103 \\
530\end{array}$ \\
\hline 4 & $\begin{array}{ll}40 & G \\
60 & F\end{array}$ & $\begin{array}{l}6,2 \\
4,2 \\
2,2\end{array}$ & $\begin{array}{r}2 \\
11 \\
128\end{array}$ & $\begin{array}{r}4 \\
46 \\
290\end{array}$ & $\begin{array}{r}8 \\
96 \\
410\end{array}$ \\
\hline 5 & $\begin{array}{ll}50 & \mathrm{G} \\
50 & \mathrm{~F}\end{array}$ & $\begin{array}{l}6,2 \\
4,2 \\
2,2\end{array}$ & $\begin{array}{r}2 \\
10 \\
115\end{array}$ & $\begin{array}{r}3 \\
34 \\
285\end{array}$ & $\begin{array}{r}7 \\
79 \\
373\end{array}$ \\
\hline 6 & $\begin{array}{l}60 \mathrm{G} \\
40 \mathrm{~F}\end{array}$ & $\begin{array}{l}6,2 \\
4,2 \\
2,2\end{array}$ & $\begin{array}{r}2 \\
6 \\
129\end{array}$ & $\begin{array}{r}3 \\
28 \\
197\end{array}$ & $\begin{array}{r}6 \\
62 \\
333\end{array}$ \\
\hline 7 & $\begin{array}{ll}70 & \mathrm{G} \\
30 & \mathrm{~F}\end{array}$ & $\begin{array}{l}6,2 \\
4,2 \\
2,2\end{array}$ & $\begin{array}{r}1 \\
6 \\
79\end{array}$ & $\begin{array}{r}1 \\
22 \\
194\end{array}$ & $\begin{array}{r}4 \\
52 \\
268\end{array}$ \\
\hline 8 & $\begin{array}{ll}80 & G \\
20 & F\end{array}$ & $\begin{array}{l}6,2 \\
4,2 \\
2,2\end{array}$ & $\begin{array}{r}1 \\
4 \\
42\end{array}$ & $\begin{array}{r}1 \\
16 \\
92\end{array}$ & $\begin{array}{r}3 \\
40 \\
171\end{array}$ \\
\hline 9 & $\begin{array}{l}90 \mathrm{G} \\
10 \mathrm{~F}\end{array}$ & $\begin{array}{l}6,2 \\
4,2 \\
2,2\end{array}$ & $\begin{array}{r}1 \\
3 \\
20\end{array}$ & $\begin{array}{r}1 \\
10 \\
53\end{array}$ & $\begin{array}{r}2 \\
24 \\
83\end{array}$ \\
\hline
\end{tabular}

G : Glucose, F : Fructose.

Im Versuch mit $50 \%$ Fructose bei $65^{\circ} \mathrm{C}$ entstehen pro Erhitzungsschritt von jeweils 10 Stunden :
1. Schritt
10 ppm HMF
2. Schritt
31 ppm HMF
3. Schritt
37 ppm HMF 

4. Schritt
51 ppm HMF
5. Schritt
62 ppm HMF

Für den entsprechenden Glucoseansatz bildet sich beim ersten Erhitzungsschritt kein HMF, bei den folgenden je $2 \mathrm{ppm}$. Die Zunahme ist faktisch linear. Bei $50 \%$ Glucose und $85^{\circ} \mathrm{C}$ ergibt sich auch bei Glucose eine exponentielle Zunahme von 13, 33, 47 und 164 ppm HMF bei 4 Erhitzungsetappen von je 10 Stunden.

\section{Saccharose}

Die HMF-Bildung aus Saccharose ist langsam, wie die Tab. 3 zeigt. Es wurden nur die niedrigen Konzentrationen angesetzt. Bei 50 Stunden, $30 \%$-iger Lösung und $65^{\circ} \mathrm{C}$ entstehen 5 ppm HMF. Unter den gleichen Bedingungen liefert Fructose $115 \mathrm{ppm}$ und Glucose 5 ppm.

ТАВ. 3. - HMF-Bildung in Saccharoselösungen bei $65^{\circ} \mathrm{C}$

TABL. 3. - HMF content in saccharose solutions at $65^{\circ} \mathrm{C}$

\begin{tabular}{|c|c|c|}
\hline $\begin{array}{c}\text { Zeit } \\
\text { (h) } \\
\text { Time }\end{array}$ & $\begin{array}{l}\text { Konzentration } \\
\text { Concentration } \\
(\%)\end{array}$ & HMF (ppm) \\
\hline 10 & $\begin{array}{l}10 \\
20 \\
30\end{array}$ & $\begin{array}{l}0 \\
0 \\
0\end{array}$ \\
\hline 20 & $\begin{array}{l}10 \\
20 \\
30\end{array}$ & $\begin{array}{l}0 \\
0 \\
0\end{array}$ \\
\hline 30 & $\begin{array}{l}10 \\
20 \\
30\end{array}$ & $\begin{array}{l}0 \\
0 \\
1\end{array}$ \\
\hline 40 & $\begin{array}{l}10 \\
20 \\
30\end{array}$ & $\begin{array}{l}1 \\
2 \\
3\end{array}$ \\
\hline 50 & $\begin{array}{l}10 \\
20 \\
30\end{array}$ & $\begin{array}{l}2 \\
4 \\
5\end{array}$ \\
\hline
\end{tabular}

\section{Maltose}

Aus Maltose entsteht vergleichsweise viel HMF, vor allem bemerkenswerterweise mehr als aus der Glucose unter den gleichen Bedingungen. In $50 \%$-igen Lösungen und bei $65^{\circ} \mathrm{C}$ wurden nach 50 -stündiger Inkubation bei der Glucose 
8 ppm HMF und bei der Maltose 28 ppm gemessen. Für Fructose ergibt sich bei identischem Ansatz 191 ppm. Die Tab. 4 unterrichtet im Detail über die Ergebnisse mit Maltoselösungen.

TAB. 4. - HMF-Bildung in Maltoselösungen bei $65^{\circ} \mathrm{C}$

TABL. 4. - HMF content in maltose solutions at $65^{\circ} \mathrm{C}$

\begin{tabular}{|c|c|c|}
\hline $\begin{array}{c}\text { Zeit } \\
\text { (h) } \\
\text { Time }\end{array}$ & $\begin{array}{c}\text { Konzentration } \\
\text { Concentration } \\
(\%)\end{array}$ & $\mathrm{HMF}(\mathrm{ppm})$ \\
\hline 10 & $\begin{array}{l}10 \\
20 \\
30 \\
40 \\
50\end{array}$ & $\begin{array}{r}0 \\
5 \\
10 \\
8 \\
15\end{array}$ \\
\hline 20 & $\begin{array}{l}10 \\
20 \\
30 \\
40 \\
50\end{array}$ & $\begin{array}{r}3 \\
7 \\
11 \\
13 \\
17\end{array}$ \\
\hline 30 & $\begin{array}{l}10 \\
20 \\
30 \\
40 \\
50\end{array}$ & $\begin{array}{r}3 \\
8 \\
15 \\
16 \\
22\end{array}$ \\
\hline 40 & $\begin{array}{l}10 \\
20 \\
30 \\
40 \\
50\end{array}$ & $\begin{array}{r}4 \\
9 \\
16 \\
19 \\
21\end{array}$ \\
\hline 50 & $\begin{array}{l}10 \\
20 \\
30 \\
40 \\
50\end{array}$ & $\begin{array}{r}5 \\
11 \\
20 \\
22 \\
28\end{array}$ \\
\hline
\end{tabular}

\section{Melezitose}

Die Versuchsergebnisse mit Melezitose sind in der Tab. 5 zusammengefaßt. Auch aus diesem Zucker kann HMF entstehen. Die Versuche wurden bei hohen Temperaturen $\left(85^{\circ} \mathrm{C}\right)$ gefahren. Die entstehenden HMF-Gehalte liegen über denen der entsprechenden Glucoselösungen. 
TAB. 5. - HMF-Bildung aus Melezitose bei $85^{\circ} \mathrm{C}$

TABL. 5. - HMF content in melezitose solutions at $85^{\circ} \mathrm{C}$

\begin{tabular}{c|c|c}
\hline $\begin{array}{c}\text { Zeit } \\
\text { (h) } \\
\text { Time }\end{array}$ & $\begin{array}{c}\text { Konzentration } \\
\text { Concentration } \\
(\%)\end{array}$ \\
& 10 & HMF (ppm) \\
5 & 20 & 2 \\
& 30 & 3 \\
50 & 4 \\
& 10 & 6 \\
\hline & 20 & 5 \\
& 30 & 10 \\
& 50 & 14 \\
& 10 & 17 \\
\hline & 20 & 26 \\
& 30 & 60 \\
& 50 & 91 \\
\hline
\end{tabular}

10. Raffinose

Schließlich wurde auch die Raffinose in die Versuche einbezogen. Die Konzentrationen mußten aus Kostengründen niedrig gehalten werden. Die Tab. 6 faßt die Resultate der Raffinose-Versuche zusammen.

TAB. 6. - HMF-Bildung aus Raffinose

TABL. 6. - HMF content in raffinose-solutions

\begin{tabular}{|c|c|c|c|}
\hline \multirow{2}{*}{$\begin{array}{c}\text { Zeit } \\
\text { (h) } \\
\text { Time }\end{array}$} & \multirow{2}{*}{$\begin{array}{c}\text { Konzentration } \\
\text { Concentration } \\
(\%)\end{array}$} & \multicolumn{2}{|c|}{ HMF (ppm) } \\
\hline & & $\begin{array}{c}\text { bei } 65^{\circ} \mathrm{C} \\
\text { at } 65^{\circ} \mathrm{C}\end{array}$ & $\begin{array}{l}\text { bei } 85^{\circ} \mathrm{C} \\
\text { at } 85^{\circ} \mathrm{C}\end{array}$ \\
\hline 10 & $\begin{array}{l}1 \\
1,5 \\
2\end{array}$ & $\begin{array}{l}0 \\
0 \\
0\end{array}$ & $\begin{array}{l}0 \\
0 \\
0\end{array}$ \\
\hline 20 & $\begin{array}{l}1 \\
1,5 \\
2\end{array}$ & $\begin{array}{l}0 \\
0 \\
0\end{array}$ & $\begin{array}{l}0 \\
0 \\
4\end{array}$ \\
\hline 30 & $\begin{array}{l}1 \\
1,5 \\
2\end{array}$ & $\begin{array}{l}0 \\
0 \\
0\end{array}$ & $\begin{array}{l}0 \\
0 \\
7\end{array}$ \\
\hline 40 & $\begin{array}{l}1 \\
1,5 \\
2\end{array}$ & $\begin{array}{l}0 \\
0 \\
0\end{array}$ & $\begin{array}{r}0 \\
0 \\
16\end{array}$ \\
\hline
\end{tabular}




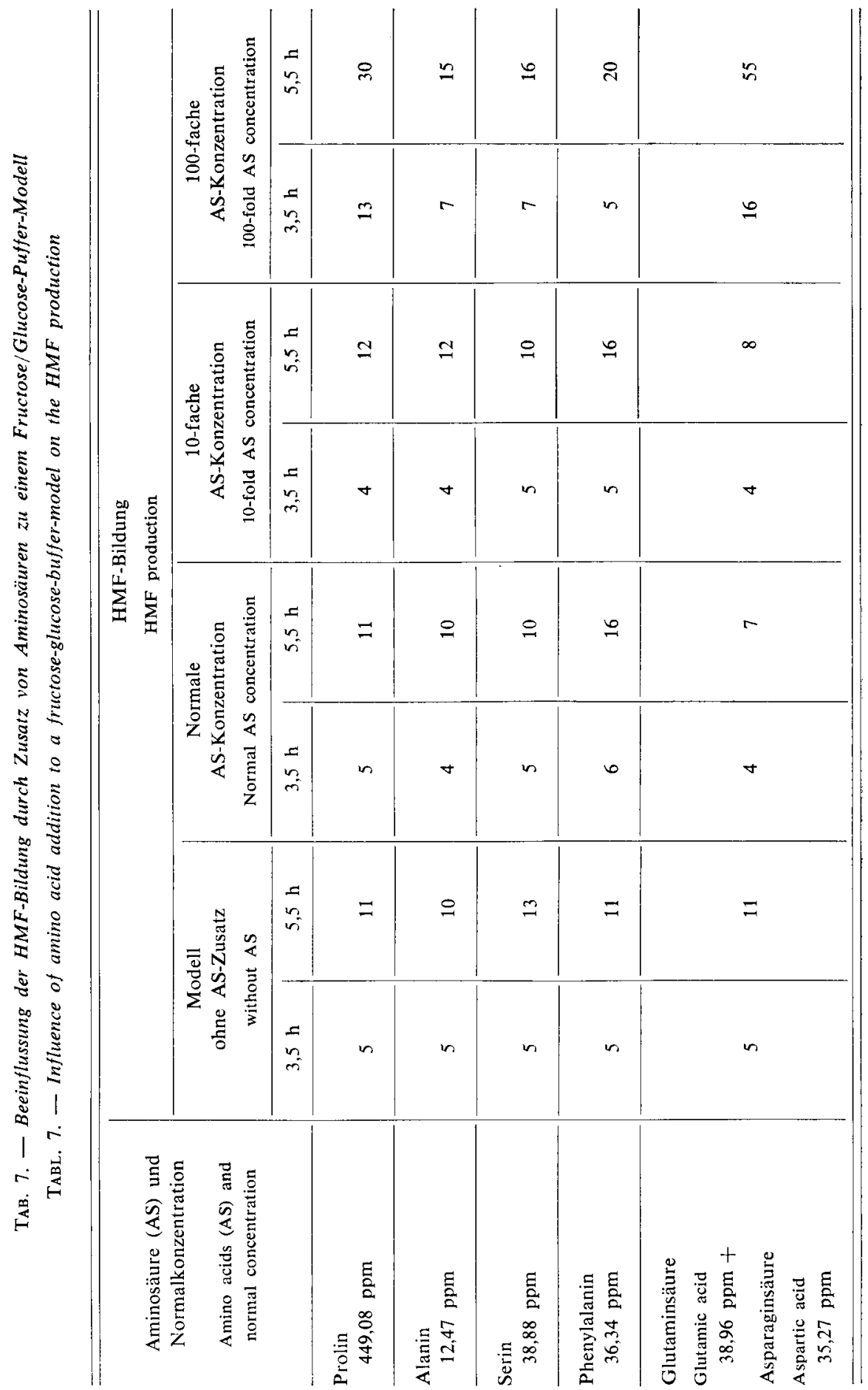




\section{Die Beeinflussung der HMF-Bildung durch Aminosäuren}

Die in Honigen mengenmäßig dominierenden Aminosäuren Prolin, Phenylalanin, Serin, Alanin, Glutamin- und Asparaginsäure wurden einer Modell-Lösung in steigenden Mengen zugesetzt. Das Modell bestand aus Fructose und Glucose zu gleichen Teilen und $20 \%$ Pufferlösung von $\mathrm{pH} 4,2$. Die Erhitzungszeit betrug 3,5 bzw. 5,5 Stunden, die Temperatur $82^{\circ} \mathrm{C}$.

Die Werte für die durchschnittlichen Aminosäuregehalte des Honigs wurden der Arbeit von BERGNer und HAHN (1972) entnommen.

Als normale Menge wurden angesetzt :

$\begin{array}{lr}\text { Prolin } & 449,08 \mathrm{mg} / \mathrm{kg} \\ \text { Alanin } & 12,47 \mathrm{mg} / \mathrm{kg} \\ \text { Serin } & 38,88 \mathrm{mg} / \mathrm{kg} \\ \text { Phenylalanin } & 36,34 \mathrm{mg} / \mathrm{kg} \\ \text { Glutaminsäure } & 38,98 \mathrm{mg} / \mathrm{kg} \\ \text { Asparaginsäure } & 35,27 \mathrm{mg} / \mathrm{kg}\end{array}$

Neben einer Versuchsreihe mit den hier angegebenen Normalgehalten wurden auch Versuche mit der 10- und 100-fachen Konzentration gefahren. Die Resultate zeigt die Tabelle 7. Bei einfacher und bei der 10-fachen Aminosäurekonzentration ist die meßbare HMF-Menge gegenüber dem Modell ohne Zusatz praktisch unverändert.

Erst bei der 100-fachen Konzentration zeigt sich bei Prolin sowie bei Glutamin- und Asparaginsäure bei längerer Erhitzung ein deutlicher Anstieg.

Werden die wichtigsten Aminosäuren der Modell-Lösung in der Normalkonzentration gleichzeitig zugegeben, so kommt es zu einer leichten Verminderung der meßbaren HMF-Menge (Tab. 8). Die auffällige Braunfärbung der Modellösungen nach der Erhitzung läßt vermuten, daß ein Teil des entstandenen HMF in Folgeprodukte der Maillard-Reaktion übergegangen ist (BAUMAnN und GiERSCHNER, 1974).

TAB. 8. - Auswirkung von Aminosäurezusatz zu einem Honigmodell bei 7-stündiger Erhitzung auf $85^{\circ} \mathrm{C}$

TABL. 8. - Influence of aminoacid addition to a honey-solution heated for 7 hours up to $85^{\circ} \mathrm{C}$

\begin{tabular}{c|c|c}
\hline \multirow{2}{*}{$\mathrm{pH}$ Wert } & \multicolumn{2}{|c}{ HMF in ppm } \\
\cline { 2 - 3 } $\mathrm{pH}$ value & $\begin{array}{c}\text { Modell ohne Aminosäuren } \\
\text { Model without AS }\end{array}$ & $\begin{array}{c}\text { Modell mit Aminosäuren } \\
\text { Model with AS }\end{array}$ \\
\hline 4,20 & 87 & 76 \\
4,10 & 86 & 81 \\
3,96 & 93 & 79 \\
\hline
\end{tabular}




\section{DISKUSSION}

Die wichtigsten im Honig vorkommenden Zucker, die in dieser Arbeit untersucht wurden, können alle zu HMF abgebaut werden, wenn auch in recht unterschiedlichem Maße.

In Übereinstimmung mit Ergebnissen zahlreicher früherer Autoren entsteht HMF besonders leicht aus Fructose, nach TäUfel und Steinbach (in Szejtli, 1976) etwa $150 \mathrm{mal}$ so schnell wie aus Glucose. In unseren Versuchen mit schwach sauren und hochkonzentrierten Modell-Lösungen bleibt das Verhältnis niedriger (maximal $40: 1$ ).

Ordnet man die von uns im Versuch geprüften Zucker nach der abnehmenden Neigung zur HMF-Bildung, so ergibt sich folgende Reihe : Fructose - Maltose Melezitose - Glucose - Saccharose.

Die Maltose wird merkwürdigerweise wesentlich leichter zu HMF abgebaut als die Glucose. In Maltose-reichen Honigen (bis zu $18 \%$ ) könnte daher ein Teil des gebildeten $\mathrm{HMF}$ auch auf den Malzzucker zurückgehen.

Erhitzt man Fructose-Glucose-Puffer-Modelle, so nimmt der HMF-Gehalt mit steigendem Fructoseanteil zu. Wenn man Modelle mit $30 \%$ Fructose und solche mit $70 \%$ Fructose in der Trockenmasse miteinander vergleicht, so bildet das Fruchtzucker-reiche Modell unter gleichen Bedingungen etwa doppelt so viel HMF wie das an Fruchtzucker-arme. 30 und $70 \%$ Fruchtzucker in der Trockenmasse entspricht etwa den Extremen, die sich bei natïrlichen Honigen finden können.

HMF-Bildung aus Glucose ist offensichtlich in schwach saurer Lösung möglich, was auch schon Diemair und JURy (1960) sowie Scarlet und Gardner (1959) festgestellt hatten, während HARWORTH und JonEs (1944) annahmen, daß dies erst im alkalischen Milieu möglich sei.

Die vielfach konstatierte Abhängigkeit der HMF-Bildung vom $\mathrm{pH}$-Wert bestätigt sich auch bei den Versuchen mit vereinfachten Honigmodellen. DiEmair und JURY (1960) beobachteteten ein Minimum der HMF-Bildung für Glucose und Fructose bei pH-Werten von 3,0-4,0. Das ließ sich unter unseren Versuchsbedingungen nicht bestätigen.

Auch in vereinfachten Honigmodellen läßt sich zeigen, daß dic HMF-Bildung in der Zeiteinheit um so größer ausfällt, je mehr HMF schon vorhanden ist. Das spricht für eine Aktivierung der HMF-Bildung durch bereits vorhandenes $\mathrm{HMF}$ und gegen die Auffassung, daß erst reaktive Zentren abgesättigt werden müßten, ehe die Akkumulierung des HMF im Honig beginnen kann. In den vereinfachten 
Modellen dürften solche reaktiven Zentren kaum vorhanden sein. Literatur zu diesem Komplex findet sich bei Hadorn und Kovacs (1960), Roman und Staub (1961), HAdorn und ZürCHER (1962) sowie bei ScHAde et al. (1958).

Bei gleicher Wärme-Belastung bildet sich in Modellen mit Zuckerkonzentrationen von 50-60\% mehr HMF als in höher konzentrierten Lösungen. Das bestätigt die Beobachtungen von HADORN et al. (1962) und ScHADE et al. (1958), wonach Honige mit hohem Wassergehalt rascher HMF bilden als wasserarme. Honig darf nach der Mehrzahl der nationalen und internationalen Bestimmungen nicht mehr als $21 \%$ (Heidehonig $23 \%$ ) Wasser enthalten.

Die niedrigsten Wassergehalte, die sich unter natürlichen Bedingungen ergeben, liegen bei $14 \%$. Insgesamt bleiben die Differenzen also unter $10 \%$. Die Modellversuche zeigen daher den Verlauf der Reaktionen klarer als die Messungen an Honigen.

Die im Honig vorhandenen freien Aminosäuren wirken leicht erniedrigend auf den meßbaren HMF-Gehalt, was mit einem durch diesen Zusatz verstärkten Weiterreagieren im Sinne der Maillard-Reaktionen zu tun haben dürfte (BAumanN und Gierschner, 1974). Die Erfahrungen der Praxis stimmen mit den Ergebnissen der Modellversuche überein. In den Aminosäure-reichen Honigtauhonigen wird weniger $\mathrm{HMF}$ gefunden als in Blütenhonigen unter den gleichen Bedingungen. Allerdings spielt dabei auch der höhere $\mathrm{pH}$-Wert der Honigtauhonige eine wichtige Rolle. Die fördernde Wirkung der Aminosäuren auf die HMF-Bildung, die z.B. von Diemaier und JURY (1960) konstatiert wurde, zeigt sich offensichtlich erst bei hohen Aminosäurekonzentrationen.

Eingegangen im April 1984.

\title{
RÉSUME \\ FACTEURS QUI INFLUENCENT LA FORMATION D'HYDROXYMETHYLFURFURAL DANS LE MIEL
}

\begin{abstract}
L'hydroxyméthylfurfural (HMF) se forme lorsque le miel est chaufé ou conservé longtemps dans une pièce chaude. Des valeurs élevées d'HMF indiquent des réactions indésirables, telles que perte ou modifications de l'arôme, diminution de l'activité bactériostatique, etc. Les normes de qualité de nombreux pays précisent les valeurs maximales admises d'HMF dans le miel. It est par conséquent intéressant de connaître les facteurs qui influencent la formation d'HMF dans des conditions déterminées. Pour obtenir des résultats plus clairs, on a travaillé avec des modèles de miel simplifiés (sucres + solution tampon de citrate-phosphate). La teneur en HMF a été mesurée par la méthode photométrique de Winkler. Parmi les facteurs étudiés figurent le type de sucre, sa concentration, la température, la durée, la valeur du $\mathrm{pH}$, l'influence de l'HMF déjà présent, les acides aminés.
\end{abstract}

La tendance à la formation d'HMF décroît lorsque l'on passe d'une solution de fructose à une solution de maltose, puis de mélézitose, de glucose et de saccharose. Toutes conditions 
identiques par ailleurs, il se forme jusqu'à 40 fois plus d'HMF dans des solutions de fructose que dans des solutions de glucose. Il existe une corrélation positive entre la formation d'HMF dans des solutions tampons de sucre et la température ou le temps et une corrélation négative avec la valeur du pH. Jusqu'à une concentration en sucres de 50-60\%, la corrélation avec la concentration est positive. Dans des solutions fortement concentrées, la quantité d'HMF formé est à nouveau plus réduite. Si l'on ajoute aux modèles de miel des acides aminés dans des concentrations semblables à celles que l'on trouve dans le miel, la quantité d'HMF mesurable diminue dans de faibles proportions. Les valeurs sont présentées en détail dans les 3 figures et les 8 tableaux.

\section{SUMMARY \\ FACTORS CONTROLLING THE PRODUCTION OF HYDROXYMETHYLFURFURAL IN HONEY}

Hydroxymethylfurfural (HMF) builds up if honey is heated or stored at warm temperature. High HMF values indicate unwanted alterations such as change and loss of aroma, and loss in bacteriostatic activity. Quality standards of many countries give maximum values for HMF. Therefore, it is of practical interest to know the factors influencing the amount of HMF produced in honey under storage conditions. In order to standardize results the authors use simplified honey models (sugars and citrate-phosphate buffer-solutions). The photometric WinkLER-method was used to measure the HMF content. Factors studied were : chemistry of the sugar including concentration, temperature, time and $\mathrm{pH}$ value; influence of $\mathrm{HMF}$ already present, and aminoacid content. There was a decreasing tendency to build up HMF in solutions of fructose-maltosemelezitose-glucose-saccharose. Under the same conditions up to 40 times more HMF was produced in fructose-solutions than in glucose-solutions. The production of $\mathbf{H M F}$ in simplified honey models is positively correlated with temperature and time, and negatively with the $\mathrm{pH}$ value. HMF production is also positively correlated with sugar concentrations up to $50-60 \%$. In solutions of higher concentration, the amount of HMF becomes lower again. The addition of amino acids (prolin, alanin, serin, phenylalanin, glutamic acid and aspartic acid) in concentrations as found in natural honeys, slightly lowers the amount of HMF available. Detailed data are given in 3 figures and 8 tables.

\section{LITERATUR}

Baumann G., Gierschner K., 1974. - Die Bedeutung aminogruppenhaltiger Verbindungen, insbesondere der freien Aminosäuren für pflanzliche Lebensmittel, vor allem für Fruchterzeugnisse. Dtsch. Lebensmittel Rundsch., 70, 273-276.

Bergner K., HahN H., 1972. - Zum Vorkommen und zur Herkunft der freien Aminosäuren im Honig. Apidologie, 3, 5-34.

Diemair W., JuRY E., 1960. - Beitrag zur Veränderlichkeit und Bildung von 5-Hydroxymethylfurfural. Z. Lebensmittelunter. Forsch., 113, 189-197.

Hadorn H., Kovacs A.S., 1960. - Zur Untersuchung und Beurteilung von ausländischen Bienenhonigen auf Grund des Hydroxymethylfurfural- und Diastasegehalts. Mitt. Lebensmittelunters. Hyg., 51, 373-390.

HADORN H., ZürRChER K., 1962. - Über die Veränderung von Bienenhonig bei der großtechnischen Abfüllung. Mitt. Lebensmittelunters. Hyg., 53, 28-34.

Hadorn H., Zürcher K., DoevelaR F.H., 1962. - Über Wärme- und Lagerschäden von Bienenhonig. Mitt. Lebensmittelunters. Hyg., 53, 191-225.

HaWORTH W.N., Jones W.G.M., 1944. - The conversion of sucrose into Furan-compounds. Part I. Hydroxymethylfurfuralaldehyd and some derivates. J. chem. Soc., 667-670. 
Jachimowicz T., Ruttner H., 1974. - Die Verwendung von Invertzucker für die Bienenfütterung anstelle von Honig. Bienenvater, 95 (3), 67-72.

Jachimowicz T., El Sherbini G., 1975. - Zur Problematik der Verwendung von Invertzucker für die Bienenfütterung. Apidologie, 6 (2), 121-143.

Lang K., Kiekebusch W., Bässler K., Griem W., CzoK L., 1981. - Untersuchungen über die Toxizität von HMF. - Disch. Lebensmittel Rundsch., 3, 98-99.

RAPEANU M.D., 1969. - Alimentary toxicosis of bees due to acid hydrolysed unrefined carbohydrates (in rumänisch). Apicultura (Rumania), 23 (4), 14-15.

Roman E., Staub M., 1961. - Hydroxymethylfurfural in Honig. Mitt. Lebensmittelunters. Hyg., 58, 44-58.

Scarlet B.L., Gardner J.G., 1959. - Formation of 5-Hydroxymethylfurfural from D-glucose in aqueous solution. J. amer. Chem. Soc., 67, 1934-1935.

SChade J.E., MARSh G.L., EckerT F.J., 1958. - Diastaseactivity and hydroxymethylfurfural in honey and their usefullnes in detection of heat adulterations. Food Res., 23, 446-463.

Szejtli J., 1976. - Säurehydrolyse glucosidischer Bindungen. VEB Fachbuchverlag, Leipzig.

Temnov V.A., 1950. - Injury to bees by caramelized honey. Proc. Lenin Acad. Agric. Science., 15 (3), $50-52$.

VorwoHi. G., 1969. - Der Hydroxymethylfurfuralgehalt in deutschen Honigen. Z. Bienenforsch., 9, 504-508.

WinkLER O., 1955. - Beitrag zum Nachweis und zur Bestimmung von Oxymethylfurfural in Honig und Kunsthonig. Z. Lebensmittelunters. Forsch., 102, 161-165. 